\title{
Bortezomib/Dexamethasone/Pegylated Liposomal Doxorubicin Regimen
}

National Cancer Institute

\section{Source}

National Cancer Institute. Bortezomib/Dexamethasone/Pegylated Liposomal

Doxorubicin Regimen. NCI Thesaurus. Code C113828.

A treatment regimen consisting of a combination of bortezomib, dexamethasone and pegylated liposomal doxorubicin. 